\title{
The Effect of Age and the Delay before Surgery on the Outcomes of Intercostal Nerve Transfers to the Musculocutaneous Nerve: A Retrospective Study of 232 Cases of Posttraumatic Total and Near-total Brachial Plexus Injuries
}

\author{
Anil Bhatia ${ }^{1}$ Aditi Kulkarni ${ }^{1} \quad$ Pablo Zancolli2,e \\ Tarek El-Gammal ${ }^{4} \quad$ Alex Muset ${ }^{5}$ \\ ${ }^{1}$ Departmentof Brachial Plexus, Deenanath Mangeshkar Hospital \\ and Research Center, Pune, Maharashtra, India \\ 2Departmentof Hand Surgery, Fundacion Favaloro, Buenos Aires, \\ Argentina \\ ${ }^{3}$ Department of Brachial Plexus and Hand Surgery, Clínica Mielina \\ Plexo Braquial, Hospital Real San José, Guadalajara, Mexico \\ ${ }^{4}$ Departmentof Brachial Plexus, Assiut University, Assiut, Egypt \\ ${ }^{5}$ Departmentof Brachial Plexus and Hand Surgery, Muset Institute, \\ Barcelona, Spain
}

Raul Rodriguez Martinez ${ }^{3}$ Jorge Clifton ${ }^{3}$

Indian J Plast Surg:2020;53:260-265

\author{
Address for correspondence Anil Bhatia, MD, Department \\ of Brachial Plexus, Deenanath Mangeshkar Hospital and \\ Research center, Erandwane, Pune 411004, Maharashtra, India \\ (e-mail: agbhatia63@gmail.com).
}

\begin{abstract}
Keywords

- brachial plexus injury

- elbow flexion

reconstruction

- intercostal nerve

transfer

- preoperative delay

Introduction Posttraumatic brachial plexus injuries are devastating, as the brain and spinal cord are disconnected from the upper limb. Restoration of elbow flexion has been widely recognized as the primary objective of nerve reconstruction. In the absence of utilizable (ruptured) root stumps in the neck, one has recourse only to nerve transfers. The direct transfer of intercostal nerves to the musculocutaneous nerve is one of the techniques that has been commonly employed over the past four decades. However, the outcomes of this procedure cited in the literature have varied considerably. The patient's age and the delay from the accident to surgery have been known to affect the results of nerve reconstruction operations. The authors present a study of the effect of these parameters on intercostal nerve transfers.

Methods The data of 232 patients with total and near-total brachial plexus injuries treated by the senior author between April 1995 and December 2015 was examined. Intercostal nerve transfers were used for the restoration of biceps function in each of these patients. The outcomes were tabulated, and the correlation with the age and the delay before surgery was examined.

Results The strength of the biceps regained was better in patients younger than 30 years old and those operated upon earlier than 6 months from the accident. The differences in outcomes were found to be statistically significant ( $p=0.001$ for preoperative delay and $p<0.005$ for the patient's age).

Conclusion The results give clear proof of the significant effect of the age and preoperative delay on the outcomes of intercostal nerve transfers for restoration of biceps function. These findings can serve as pointers to help the surgeon in choosing the method of nerve reconstruction in a given case.
\end{abstract}




\section{Introduction}

Brachial plexus lesions result in a disconnection of the upper limb nerves from the spinal cord and brain. Surgery aims to restore function in some of the paralyzed muscles to improve the control of the flail limb. Elbow flexion has been recognized as the most important function to be restored in adults in this type of injury. ${ }^{1,2}$ This function can be achieved through the reinnervation of elbow flexors from ruptured roots suitable for grafting or through nerve transfers. ${ }^{3}$ When possible, intraplexual nerve transfers are preferred. In total or near-total brachial plexus injuries, we are usually forced to resort to using extraplexual donor nerves. These include the intercostal nerves, the spinal accessory nerve, the phrenic nerve, and contralateral C7 root..$^{4-6}$ Intercostal nerves have been used to target the lateral cord, the musculocutaneous nerve, or even the biceps nerve branch. ${ }^{7-9}$ Direct intercostal nerve transfer to musculocutaneous nerve is recommended, avoiding interpositional grafts for better functional outcomes..$^{10}$ The use of 3 or 4 intercostal nerves (third to sixth) provides a good and direct matching to the musculocutaneous nerve. ${ }^{11,12}$ Unit across the world have reported varying outcomes of intercostal nerves transfer for elbow flexion. ${ }^{13-17}$ Hence, it is difficult to adopt a benchmark for the expected result in a given patient. Prediction of the outcome is important, as that dictates the choice of technique employed for restoration of elbow flexion. It is known that the patient's age and the delay prior to surgery are the two main parameters affecting the outcome. To our knowledge, there is limited data published on the influence of age on the results of nerve reconstruction. In general, surgery is performed when we are sure that spontaneous recovery is not possible. Hence, the accepted delay in most cases is a minimum of a month and has commonly been suggested to be 3 to 6 months. It is important to know our limits, beyond which a successful outcome cannot be reasonably expected. That would determine the delay at which one would be forced to opt for a salvage procedure (free microvascular muscle transfer) that is more demanding, takes longer to perform, and involves use of greater resources. The objective of this study was to determine the probability of successful restoration of elbow flexion using intercostal nerve transfers to the musculocutaneous nerve in posttraumatic total brachial plexus injuries and figure the limitations of this procedure. The strength of the biceps restored was measured in a series of patients operated by one surgeon (the senior author A.B.). The outcomes were correlated with the patient's age and the delay between the accident and the surgery. The authors proposed to use this information to formulate guidelines for the treatment of posttraumatic total brachial plexus injures.

\section{Materials and Methods}

The records of patients operated upon by the senior author were examined. We excluded patients in whom follow-up data of 12 months was unavailable. Demographic information regarding age at surgery and gender was recorded. We analyzed data, including type of injury, delay between the injury and surgery, types of procedures performed and follow-up intervals, and recorded the results in terms of strength regained in the target muscles at those follow-up intervals. This study was limited to evaluation of the biceps in patients in whom intercostal nerves had been transferred to the musculocutaneous nerve in total palsies (flail upper limbs) and near-total brachial plexus injuries. A successful outcome was noted when the patient could flex the elbow to 90 degrees independently with $1 \mathrm{~kg}$ weight tied at the wrist. The data for success of each procedure was correlated with the age of the patient and the time interval between the accident and the surgical reconstruction.

\section{Statistical Analysis}

Data was tabulated into MS Excel, and analysis was performed using the SPSS 20.0 for Windows. The description of the data was done in form of arithmetic mean + /-standard deviation (SD) for quantitative data, while in the form of frequencies (\%) for qualitative (categorical) data. $p$ values of $<0.05$ were considered as significant. For comparison of categorical variables (i.e., to examine the associations between qualitative/quantitative variables), chi-square test is used, if the number of elements in each cell are 5 or higher and Fisher's exact test otherwise.

\section{Procedure}

The musculocutaneous nerve was isolated distal to the pectoralis major insertion. It was traced as proximally as possible, divided, and turned to the axilla. The chest wall was exposed by extending the incision along the sixth rib, and three intercostal nerves were harvested (usually the 4 th to the 6th) by stripping the serratus anterior, intercostal muscles and the periosteum from the lower margin of each rib. If the nerve was found injured due to previous fractures of the ribs or passage of intercostal drains, the adjacent level was exposed to complete the necessary quota of three intercostals. In each case, the intercostal nerves were directly approximated to the musculocutaneous nerve using $9 / 0$ or $10 / 0$ nylon sutures and the repairs were, sometimes, reinforced with glue. The limb was supported in adduction across the chest in an arm pouch sling for a month. Passive mobilization of the shoulder was limited to 30 degrees for another month. The patients were taught passive mobilization of the elbow, and galvanic stimulation of the biceps muscle was used while awaiting signs of reinnervation of the biceps. Early contraction of the biceps was usually seen on straining (lifting the head while lying supine) at 6 to 7 months after surgery. This was demonstrated to the patient and his/her attendants, and they were instructed in methods to strengthen the biceps. Progress to grade 3 function (ability to flex the elbow while standing) depended upon the patient's diligence in performing the strengthening exercises. The patients were reviewed at intervals of 2 to 3 months till a plateau in the progress of the biceps was noted. A successful outcome was 
noted when the patient could flex the elbow 90 degrees or more independently with $1 \mathrm{~kg}$ weight tied at the wrist.

\section{Results}

A total of 524 patients were analyzed. In 232 patients of these, three intercostal nerves were used to reinnervate the biceps through a nerve transfer to the musculocutaneous nerve. The demographic characteristics of patients are shown in - Table 1. Ninety-five percent were male. The average age of patient was $30.26 \pm 9.486$ years. The average duration for delay in surgery was $135.17 \pm 102.6$ days and the average follow-up duration was $30.45 \pm 21.6$ months. These included 200 patients of total palsies (flail upper limbs) and 32 extended partial palsies. The outcomes in patients with flail upper limbs were studied. They were also compared with the corresponding figures in the group of partial palsies.

\section{Statistical Analysis \\ Association between the Delay from the Accident and the Biceps Outcomes in Total Palsies}

In 200 patients with total brachial plexus injuries, three intercostal nerves were transferred to the main trunk of the musculocutaneous for restoration of the biceps. We found that patients who underwent surgery within 180 days had a successful outcome (MRC grade 3 and above) more often than patients who underwent surgery at a longer delay (73.9\% vs. 46.5\%). This difference between patients operated upon beyond 6 months was found to be statistically significant $(p=0.001)(-$ Table 2 and $\boldsymbol{- F i g . ~} \mathbf{1})$.

Table 1 Demographic characteristics: $(n=232)$

\begin{tabular}{|l|l|}
\hline Characteristics & $\boldsymbol{n}(\%)$ \\
\hline Gender & \\
\hline Male & $222(95.7)$ \\
\hline Female & $10(4.3)$ \\
\hline Age & \\
\hline Mean \pm SD & $30.39 \pm 9.8$ years \\
\hline$<30$ years & $131(56.5)$ \\
\hline $30-40$ years & $61(26.3)$ \\
\hline$>40$ years & $40(17.2)$ \\
\hline Delayed before surgery (in days) & \\
\hline Mean \pm SD & $128.94 \pm 94.4$ \\
\hline$<180$ & $183(78.9)$ \\
\hline $180-240$ & $21(9.1)$ \\
\hline$\geq 240$ & $28(12.1)$ \\
\hline Postoperative biceps grade (MRC) & \\
\hline 0 & $19(8.2)$ \\
\hline 1 & $25(10.8)$ \\
\hline 2 & $32(13.8)$ \\
\hline$\geq 3$ & $156(67.2)$ \\
\hline
\end{tabular}

Abbreviation: MRC, Medical Research Council.
Association between the Age at Surgery and the Biceps Outcomes in Total Palsies

We found a significant association $(p<0.05)$ between age and the strength of the biceps regained in patients with total brachial plexus injuries who underwent three intercostal nerves transfer to musculocutaneous. Patients younger than 30 years old had better outcomes (Medical Research Council [MRC] grade 3 and above) than older patients. The percentage of a successful outcome (MRC grade 3 and greater) in patients younger than 30 years was significantly better $(81.4 \%)$ than in older patients (48.8\%). ( - Table 3 and $\boldsymbol{- F i g . ~ 2 ) . ~}$

These observations were also found to apply to the group of 32 patients with extended partial palsies in whom intercostal nerve transfers to the musculocutaneous nerve were performed. The differences in outcomes were statistically significant in these patients as well ( - Tables 4 and 5 ).

\section{Discussion}

Total adult brachial plexus injuries are devastating injuries. Elbow flexion is widely recognized as the most important function to be restored in these patients. In the absence of utilizable roots stumps in the neck, one has recourse only to nerve transfers for reinnervation of the biceps. In extensive partial palsies, the residual hand function is often insufficient to allow intraplexual transfer using fascicles of the functioning median/ulnar nerves. In total palsies, one has to utilize options outside the brachial plexus. A variety of nerve donors have been described. Use of the hypoglossal nerve was proposed in the 1970s and 1980s. Kotani, and subsequently, Alieu, described the use of the spinal accessory nerve bridged to the musculocutaneous nerve using a nerve graft. Nagano (1989) and Chuang (1993) described their experience using intercostal nerves by direct transfer to the musculocutaneous nerve. ${ }^{11,12}$ Direct transfers are preferred as the interposition of a nerve graft inevitably leads to a delay in reinnervation, a drop in the success rate and, eventually, weaker biceps. Use of the contralateral C7 for restoration of two functions (biceps and fingers/wrist flexion) has been practiced since $1986,{ }^{4}$ but the method is not universally accepted, and fear of producing a deficit in the donor limb has prevented harvest of the full $\mathrm{C}$. This has resulted in poorer outcomes and greater disillusionment with that transfer. The patient's age and the delay since the accident have been noted to be the two main parameters that affect the outcomes of nerve reconstruction. However, statistical proof has not been provided for the efficiency of individual procedures that could guide the strategy in a given patient. Jivan et $\mathrm{a}^{18}$ have referred specifically to the effect of the delay between the accident and the surgical reconstruction upon the outcome. However, the authors were building consensus for exploration within a week from the injury, and nerve grafting was the reconstructive option evaluated. Ruch et $\mathrm{al}^{19}$ suggest that, theoretically, the overall success rate could be improved performing the neurotization of intercostals to elbow flexion with an interval from the injury to surgery less than 5 months in patients under 50 years old. Xiao et $\mathrm{al}^{20}$ noted statistically significant difference of useful elbow flexion in patients under 40 years 
Effect of Age and the Delay before Surgery on the Outcomes of Intercostal Nerve Transfers Bhatia et al. 263

Table 2 Association between the delay before the operation and the biceps outcomes in total palsies $(n=200)$

\begin{tabular}{|l|l|l|l|}
\hline \multirow{2}{*}{ Delay in surgery in days } & \multicolumn{2}{|c|}{ Biceps grades at follow-up } & Chi-square value $(p)$ \\
\cline { 2 - 3 } & MRC 0 to 2 & MRC 3 and 4 & \multirow{2}{*}{$11.624(p=0.001)$} \\
\hline$<180(n=157)$ & $41 / 157(26.1 \%)$ & $116 / 157(73.9 \%)$ & \\
\hline$>180(n=43)$ & $23 / 43(53.5 \%)$ & $20 / 43(46.5 \%)$ & \\
\hline
\end{tabular}

Abbreviation: MRC, Medical Research Council.

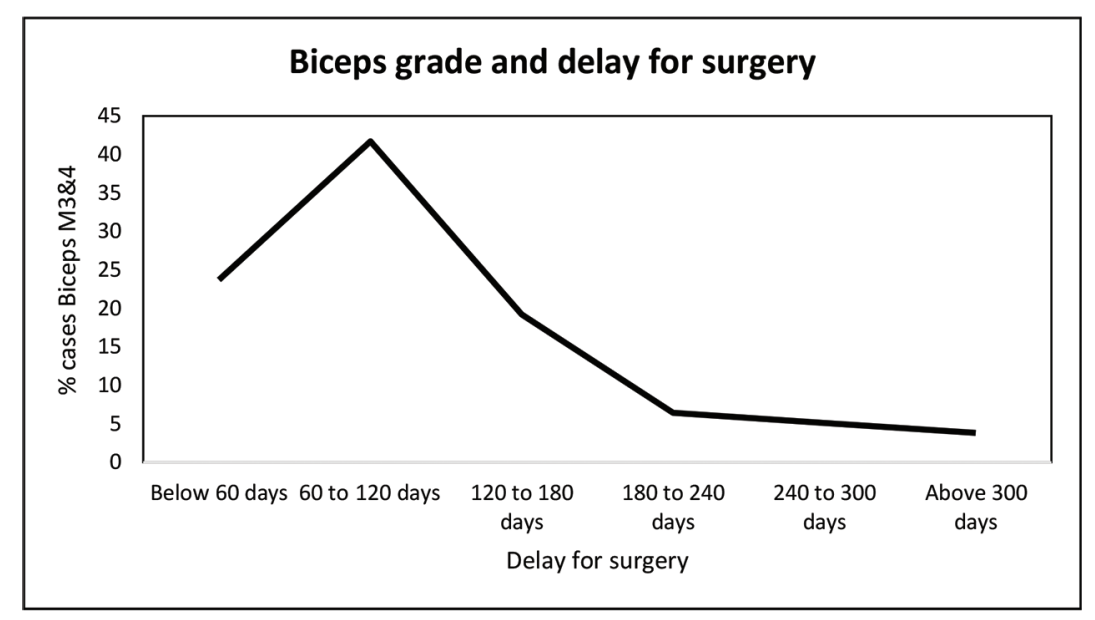

Fig. 1 Percentage of cases with biceps grade M3 or $>$ and time delay for surgery $(n=200)$.

Table 3 Association between the age of the patients and the biceps outcomes in total palsies $(n=200)$

\begin{tabular}{|l|l|l|l|}
\hline \multirow{2}{*}{ Age at surgery } & \multicolumn{2}{|c|}{ Biceps grades at follow-up } & Chi-square value $(p)$ \\
\cline { 2 - 3 } & $\mathbf{0}$ to 2 & $\mathbf{3}$ and 4 & \multirow{2}{*}{$23.59(p<0.05)$} \\
\hline$<30$ years $(\boldsymbol{n}=\mathbf{1 1 8})$ & $22 / 118(18.6)$ & $96 / 118(81.4)$ & \\
\hline$>30$ years $(\boldsymbol{n}=\mathbf{8 2})$ & $42 / 82(51.2)$ & $40 / 82(48.8)$ & \\
\hline
\end{tabular}

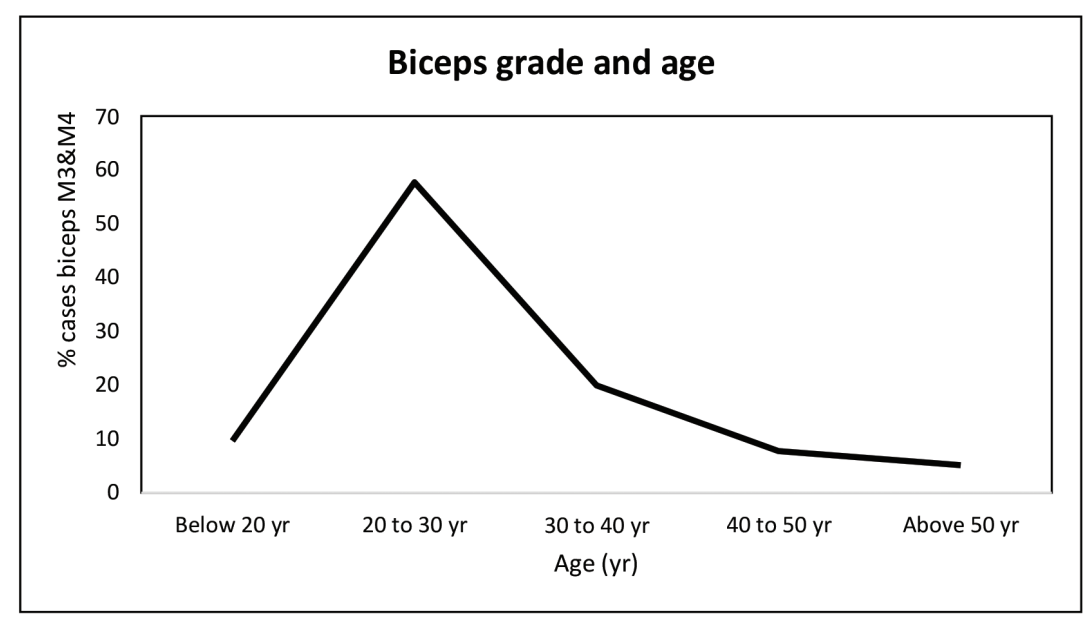

Fig. 2 Percentage of cases with biceps grade M3 or $>$ and patient age $(n=200)$.

old who received the intercostal neurotization but found no difference of recovery if the preoperative delay was within or after 6 months from the injury. However, that study was not of adequate power, as it referred to a small number of cases (30 patients). Socolovsky et al have published a study that specifically comments on the adverse influence of age 
Table 4 Association between delay before the operation and the biceps outcomes for intercostal nerve transfers in combined total and partial palsies $(n=232)$

\begin{tabular}{|l|l|l|l|}
\hline \multirow{2}{*}{ Delay in surgery in days } & \multicolumn{2}{|c|}{ Biceps grades at follow-up } & \multirow{2}{*}{ Chi-square value $(p)$} \\
\cline { 2 - 3 } & $\mathbf{0}$ to 2 & 3 and 4 & \\
\hline$<180(n=183)$ & $51 / 183(27.9 \%)$ & $132 / 183(72.1 \%)$ & $9.405(p=0.002)$ \\
\hline$>180(n=49)$ & $25 / 49(51.0 \%)$ & $24 / 49(49.0 \%)$ & \\
\hline
\end{tabular}

Table 5 Association between the age of the patient and the biceps outcome for intercostal nerve transfers in combined total and partial palsies ( $n=232$ patients)

\begin{tabular}{|l|l|l|l|}
\hline \multirow{2}{*}{ Age at surgery } & \multicolumn{2}{|c|}{ Biceps grades at follow-up } & \multirow{2}{*}{ Chi-square value $(p)$} \\
\cline { 2 - 3 } & 0 to 2 & 3 and 4 & \multirow{2}{*}{$22.77(p<0.005)$} \\
\hline$>30$ years $(n=131)$ & $26 / 131(26.1 \%)$ & $105 / 131(80.2 \%)$ & \\
\hline$>30$ years $(n=101)$ & $50 / 101(49.5 \%)$ & $51 / 101(50.5 \%)$ & \\
\hline
\end{tabular}

on the outcomes of brachial plexus reconstruction. Their study referred to patients with different patterns of injuries (total as well as partial palsies) as well as a variety of different methods used for reconstruction of elbow flexion. ${ }^{21}$

This is a study of one nerve transfer (intercostal to musculocutaneous) done in a large series of patients by the same surgeon. One outcome was measured, that is, independent elbow flexion while standing (without any help of the opposite limb) with 1 kilogram weight tied at the wrist. So, the label of grade 3 in this study cannot be compared with earlier series, where grade 3 function was deemed unsatisfactory. In the treatment of extensive palsies (total and near-total palsies), one has to utilize the best donor option for biceps restoration. The choice has to be guided by knowledge about the efficiency of each procedure. This study aims to provide such data for intercostal nerve transfers to musculocutaneous. It is difficult to provide rigid guidelines that can be adhered to in every patient. Nevertheless, the authors believe that such information is useful to improve functional reconstruction outcomes. This study shows that the outcomes for elbow flexion are better when the surgery is performed in total palsies in a patient under 30 years old and before 180 days (6 months) from the time of the injury and the difference is statistically significant. Poor results were noted when the surgery was performed later than 180 days ( 6 months) from the accident (biceps $M R C<3 ; 53.5 \%$ ) and in patients over 30 years old (biceps MRC <3; 51.2\%) in total brachial plexus injuries. Thus, in a patient older than 30 years presenting at a delay of, for instance, 7.5 months, a free muscle transfer would, perhaps, be preferable to an attempt at conventional nerve reconstruction.

In this context, the authors also wish to expand the relevance of this study. Given that transfer of intercostal nerves to the musculocutaneous nerve involves approximation of healthy nerve ends and only one outcome is measured (elbow flexion), one wonders if these results can indicate the probability of a successful nerve repair in general. A large number of patients studied negate the effect of bias created by the retrospective nature of this report, and the findings limited to the experience of one surgeon. The study provides the probability of a successful outcome in a given patient, according to patients' age and time from the injury.

\section{Conclusion}

Direct transfer of three intercostal nerves to the musculocutaneous nerve has been found to restore independent elbow flexion consistently in our series of 232 patients with total and near-total brachial plexus injuries. The outcomes were better in patients younger than 30 years, and when the surgery was performed before 6 months from the accident. The difference was found to be statistically significant. This data could influence the shift to a salvage procedure (free functioning muscle transfer) that is more efficient when an older patient presents at a delay longer than 6 months. Also, this information could prove valuable at the time of counselling the patient.

\section{Declaration}

- No source of financial and material support.

- No support provided.

- No content of this paper has been presented previously.

\section{Conflict of Interest}

None declared.

\section{References}

1 Shin AY, Spinner RJ, Steinmann SP, Bishop AT. Adult traumatic brachial plexus injuries. J Am Acad Orthop Surg 2005;13(6):382-396

2 Brophy RH, Wolfe SW. Planning brachial plexus surgery: treatment options and priorities. Hand Clin 2005;21(1):47-54

3 Sulaiman OA, Kim DD, Burkett C, Kline DG. Nerve transfer surgery for adult brachial plexus injury: a 10-year experience at Louisiana State University. Neurosurgery 2009;65(4, Suppl):A55-A62

$4 \mathrm{Gu} \mathrm{YD,} \mathrm{Zhang} \mathrm{GM,} \mathrm{Chen} \mathrm{DS,} \mathrm{et} \mathrm{al.} \mathrm{Cervical} \mathrm{nerve} \mathrm{root}$ transfer from contralateral normal side for treatment of brachial plexus root avulsions. Chin Med J (Engl) 1991;104(3):208-211 
5 Waikakul S, Wongtragul S, Vanadurongwan V. Restoration of elbow flexion in brachial plexus avulsion injury: comparing spinal accessory nerve transfer with intercostal nerve transfer. J Hand Surg Am 1999;24(3):571-577

6 Liu Y, Lao J, Zhao X. Comparative study of phrenic and intercostal nerve transfers for elbow flexion after global brachial plexus injury. Injury 2015;46(4):671-675

7 Seddon HJ. Nerve grafting.JBone JointSurg Br 1963;45:447-461

8 Narakas A. Surgical treatment of traction injuries of the brachial plexus. Clin Orthop Relat Res 1978; (133):71-90

9 Tsuyama N, Hara T. Intercostal nerve transfer in the treatment of brachial plexus injury of root avulsion type. Exerpta Medica 1972;29(1):35

10 Merrell GA, Barrie KA, Katz DL, Wolfe SW. Results of nerve transfer techniques for restoration of shoulder and elbow function in the context of a meta-analysis of the English literature. J Hand Surg Am 2001;26(2):303-314

11 Chuang DC, Yeh MC, Wei FC. Intercostal nerve transfer of the musculocutaneous nerve in avulsed brachial plexus injuries: evaluation of 66 patients. J Hand Surg Am 1992;17(5):822-828

12 Nagano A, Tsuyama N, Ochiai N, Hara T, Takahashi M. Direct nerve crossing with the intercostal nerve to treat avulsion injuries of the brachial plexus. J Hand Surg Am 1989;14(6):980-985

13 Samardzić M, Rasulić L, Grujicić D, Milicić B. Results of nerve transfers to the musculocutaneous and axillary nerves. Neurosurgery 2000;46(1):93-101, discussion 101-103 [discussion: 101-3]
14 Bhatia A, Shyam AK, Doshi P, Shah V. Nerve reconstruction: A cohort study of 93 cases of global brachial plexus palsy. Indian J Orthop 2011;45(2):153-160

15 Moiyadi AV, Devi BI, Nair KP. Brachial plexus injuries: outcome following neurotization with intercostal nerve. J Neurosurg 2007;107(2):308-313

16 Terzis JK, Barbitsioti A. Primary restoration of elbow flexion in adult post-traumatic plexopathy patients. J Plast Reconstr Aesthet Surg 2012;65(1):72-84

17 El-Gammal TA, Fathi NA. Outcomes of surgical treatment of brachial plexus injuries using nerve grafting and nerve transfers. J Reconstr Microsurg 2002;18(1):7-15

18 Jivan S, Kumar N, Wiberg M, Kay S. The influence of pre-surgical delay on functional outcome after reconstruction of brachial plexus injuries. J Plast Reconstr Aesthet Surg 2009;62(4):472-479

19 Ruch DS, Friedman A, Nunley JA. The restoration of elbow flexion with intercostal nerve transfers. Clin Orthop Relat Res 1995; (314):95-103

20 Xiao C, Lao J, Wang T, Zhao X, Liu J, Gu Y. Intercostal nerve transfer to neurotize the musculocutaneous nerve after traumatic brachial plexus avulsion: a comparison of two, three, and four nerve transfers. J Reconstr Microsurg 2014;30(5):297-304

21 Socolovsky M, di Masi G, Bonilla G, Lovaglio AC, López D. Age as a predictor of long term results in patients with brachial plexus palsies undergoing surgical repair. Oper Neurosurg (Hagerstown) 2018;15(1):15-24 